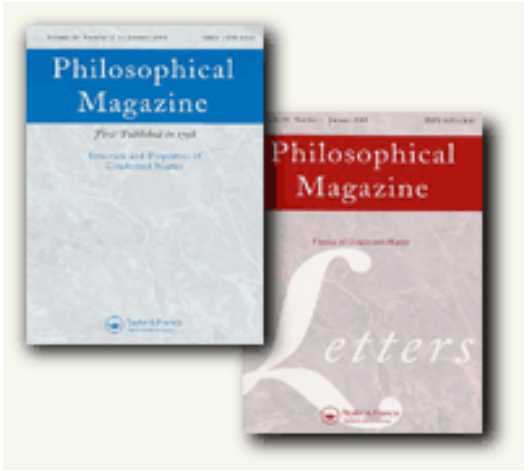

\title{
Ternary Fe-Cu-Ni many-body potential to model reactor pressure vessel steels: First validation by simulated thermal annealing
}

\begin{tabular}{|c|c|}
\hline Journal: & Philosophical Magazine \& Philosophical Magazine Letters \\
\hline Manuscript ID: & TPHM-09-May-0221.R1 \\
\hline Journal Selection: & Philosophical Magazine \\
\hline $\begin{array}{r}\text { Date Submitted by the } \\
\text { Author: }\end{array}$ & 28-Aug-2009 \\
\hline Complete List of Authors: & $\begin{array}{l}\text { Bonny, Giovanni; SCK-CEN, Nuclear Materials Science Institute } \\
\text { Pasianot, Roberto; CAC-CNEA, Materials Department } \\
\text { Castin, Nicolas; SCK-CEN, Nuclear Materials Science Institute } \\
\text { Malerba, Lorenzo; SCK-CEN, Nuclear Materials Science Institute }\end{array}$ \\
\hline Keywords: & $\begin{array}{l}\text { atomistic simulation, Fe-based alloys, interatomic potential, } \\
\text { nanoscale precipitates, structural materials }\end{array}$ \\
\hline Keywords (user supplied): & reactor pressure vessel steels \\
\hline
\end{tabular}

\section{S) ScholaroNE \\ Manuscript Central}


Ternary Fe-Cu-Ni many-body potential to model reactor pressure vessel steels: First validation by simulated thermal annealing

\author{
G. Bonny ${ }^{1,2, *}$, R.C. Pasianot ${ }^{3,4,5}$, N. Castin ${ }^{1,6}$ and L. Malerba ${ }^{1}$ \\ ${ }^{1}$ SCK $•$ CEN, Nuclear Materials Science Institute, Boeretang 200, B-2400 Mol, Belgium \\ ${ }^{2}$ Ghent University, Center for Molecular Modeling, Proeftuinstraat 86, B-9000 Gent, \\ Belgium \\ ${ }^{3}$ CAC-CNEA, Departamento de Materiales, Avda. Gral. Paz 1499, 1650 San Martín, \\ Argentina \\ ${ }^{4}$ CONICET, Avda. Rivadavia 1917, 1033 Buenos Aires, Argentina \\ ${ }^{5}$ Instituto Sábato, UNSAM/CNEA, Avda. Gral. Paz 1499, 1650 San Martín, Argentina \\ ${ }^{6}$ Université Libre de Bruxelles (ULB), Physique des Solides Irradiés et des Nanostructures (PSIN), \\ Bd. du Triomphe CP234, 1050 Brussels, Belgium.
}

\begin{abstract}
In recent years the development of atomistic models dealing with microstructure evolution and subsequent mechanical property change in reactor pressure vessel steels has been recognised as an important complement to experiments. In this framework, a literature study has shown the necessity of many-body interatomic potentials for multi-component alloys. In this paper we develop a ternary many-body $\mathrm{Fe}-\mathrm{Cu}-\mathrm{Ni}$ potential for this purpose. As a first validation, we used it to perform a simulated thermal annealing study of the $\mathrm{Fe}-\mathrm{Cu}$ and $\mathrm{Fe}$ $\mathrm{Cu}-\mathrm{Ni}$ alloys. Good qualitative agreement with experiments is found, although fully quantitative comparison proved impossible, due to limitations in the used simulation techniques. These limitations are also briefly discussed here.
\end{abstract}

\title{
1. Introduction
}

In recent years the development of models tracing the microstructure evolution and subsequent mechanical property change in reactor pressure vessel (RPV) steels, employing a computer-based multi-scale approach, has been recognised as an important complement to

* Corresponding author, email: gbonny@ sckcen.be 
experiments, in support of the surveillance programmes of existing nuclear power plants (NPPs) [1]. The development of physical models of this type is considered particularly important in view of the extension of the service lifetime of NPPs, as for this purpose the available database from surveillance specimens is not sufficient and solid models suitable to be extrapolated are therefore required in order to prove the capability of the materials to perform their duty for a time twice as long than foreseen.

RPV steels used in Western-type light water reactor NPPs are low-alloyed bainitic steels, containing as main alloying elements nickel, manganese and often molybdenum, as well as consistent traces of silicon, copper and, to a lesser extent, chromium, sulphur and phosphorus. The low solubility of copper in iron leads to radiation-enhanced formation of ultrafine copper-rich precipitates during operation at $290^{\circ} \mathrm{C}$, which atom probe studies have revealed to contain nickel, manganese, silicon, and sometimes phosphorus as well [2-4]. Even in low-copper steels, precipitates rich in manganese and nickel are detected, while phosphorus atoms have in all cases the tendency to distribute in a heterogeneous manner, associated or not with other precipitates [4-6]. The formation of these different types of precipitates may be at least partly radiation-induced, but no consensus exists about their actual origin, nature and mechanism of formation. There is, however, consensus about the fact that the interaction of these nano-defects with dislocations is the main cause of hardening and embrittlement of these steels [7-16]. In this framework, large scale atomistic simulations in multi-component alloys are of fundamental importance with a view to cast some light on the mechanisms leading to the formation of the mentioned different classes of precipitates, as well as in order to study in detail their interaction with dislocations as source of hardening.

In the literature a few works studying precipitation in multi-component alloys representing RPV steels exist. For example, in the works of Liu et al. [17] and Odette and Wirth [18], precipitation in the Fe-Cu-Ni-Mn-Si model alloy is studied by means of rigid lattice exchange Monte Carlo (MC). Therein the atomic interactions were parameterized by approximate pair bond potentials derived from thermodynamic data. However, simple pair potentials are inadequate to treat diffusion and cannot correctly describe defect configurations (see $[19,20]$ and references therein). Therefore they are inadequate to trace the microstructural evolution of the alloy in real time and offer limited transferability to off-lattice atomistic methods, for example molecular dynamics (MD). Other works include the ones by Vincent et al. [21, 22], where rigid lattice kinetic MC was performed on the same model alloy. In those works the interatomic interactions were parameterized by pair interactions based on density functional theory (DFT) calculations. Also here the interatomic interactions 
are not transferrable to off-lattice approaches, thereby limiting the applicability of the model. From these works it is clear that more realistic and extendable simulations (including offlattice approaches, allowing for example also dislocation-precipitate interaction studies) require the development of reliable multi-component many-body interatomic potentials.

In this framework we present a many-body interatomic potential for a ternary alloy, namely $\mathrm{Fe}-\mathrm{Cu}-\mathrm{Ni}$, developed in such a way that both the alloy's thermodynamic properties and the interaction between solute atoms and point-defects is properly reproduced, as compared to, respectively, the experimental phase diagram and density functional theory (DFT) calculations. The choice of the Fe-Cu-Ni alloy as first step towards the development of potentials for multi-component systems was dictated by the fact that these two alloying elements are phenomenologically considered to be the main responsibles for RPV steel hardening and embrittlement $[13,23]$.

As a first validation of our potential, we carried out an atomistic study regarding precipitation in $\mathrm{Fe}-\mathrm{Cu}$ and $\mathrm{Fe}-\mathrm{Cu}-\mathrm{Ni}$ alloys, to determine the effect of $\mathrm{Ni}$ on $\mathrm{Cu}$ precipitate formation. For this purpose, we used molecular static (MS) calculations to characterize $\mathrm{Cu}$ precipitate/Ni interaction and atomistic kinetic Monte Carlo (AKMC) to trace the microchemical evolutions of the alloy.

\section{Methodology}

\section{Energy formalism}

Formatted: Bullets and Numbering

The atomic interactions are described using the embedded atom method (EAM) [24], which is widely used to describe metals and their alloys. In addition to pair interactions, $V$, this approach includes an embedding energy, $F$, dependent on the local electron density, $\rho$. The latter contribution approximates the many-body contribution of all nearby atoms. The total energy within EAM is given as,

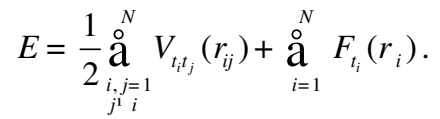

Here $N$ represents the total number of atoms in the system, $r_{i j}$ is the distance between atoms $i$ and $j$, and $t_{i}$ denotes the atom-type ( $\mathrm{Fe}, \mathrm{Cu}$ or $\mathrm{Ni}$ in our case). The local electron density around atom $i$, contributed from its neighbours is given as, 


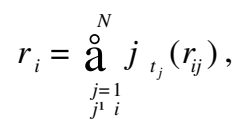

where $\rho$ denotes the electron density function of the considered element. Thus, for the $\mathrm{Fe}-\mathrm{Cu}-$ Ni ternary system twelve functions need to be defined: $\varphi_{\mathrm{Fe}}, \varphi_{\mathrm{Cu}}, \varphi_{\mathrm{Ni}}, F_{\mathrm{Fe}}, F_{\mathrm{Cu}}, F_{\mathrm{Ni}}, V_{\mathrm{FeFe}}$, $V_{\mathrm{CuCu}}, V_{\mathrm{NiNi}}, V_{\mathrm{FeCu}}, V_{\mathrm{FeNi}}$ and $V_{\mathrm{CuNi}}$.

The pure species functions are taken from the literature, choosing the most suitable among those currently available. For Fe we choose 'potential 2' developed by Mendelev et al. [25], for $\mathrm{Cu}$ we choose 'EAM 1' developed by Mishin et al. [26] and for Ni we adopted the potential developed by Voter and Chen [27]. Within EAM, however, the forms of $V, F$ and $\varphi$ are not uniquely determined. Two transformations $[19,28,29], \hat{T}_{1}$ and $\hat{T}_{2}$, exist that leave the total energy invariant,

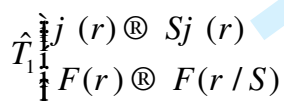

$$
\begin{aligned}
& \begin{array}{c}
\hat{T}_{2} \frac{1}{\mathrm{i}} F(r) \circledR F(r)+C r \\
\mathrm{~A} V(r) \circledR V(r)-2 C j(r)
\end{array},
\end{aligned}
$$

with $C$ and $S$ arbitrary constants. Therefore the units of the electron density are arbitrary for the pure species, but contribute to each others' embedding energy in the alloy case. To assure a priori an equal contribution to the electron density from all elements, we use transformations $\hat{T}_{1}$ and $\hat{T}_{2}$ with $C=-F^{\prime}\left(\rho_{\mathrm{eq}}\right)$ and $S=1 / \rho_{\mathrm{eq}}$ to transform the pure potentials to their "effective gauge" form [19], where $F^{\prime}\left(\rho_{\mathrm{eq}}\right)=0$ and $\rho_{\mathrm{eq}}=1$ on the bcc equilibrium lattice.

Following this, the three cross interactions $V_{\mathrm{FeCu}}, V_{\mathrm{FeNi}}$ and $V_{\mathrm{CuNi}}$ and the relative weight between the electron densities $\rho_{\mathrm{Fe}} / \rho_{\mathrm{Cu}}, \rho_{\mathrm{Fe}} / \rho_{\mathrm{Ni}}$ need to be determined. The cross pair potentials $V_{\mathrm{FeCu}}, V_{\mathrm{FeNi}}$ and relative weights between the electron densities $\rho_{\mathrm{Fe}} / \rho_{\mathrm{Cu}}, \rho_{\mathrm{Fe}} / \rho_{\mathrm{Ni}}$ are taken from the works by Pasianot and Malerba [30] and Bonny et al. [31], respectively. Therefore, our fitting effort is focussed on $V_{\mathrm{CuNi}}$. In all cases the cross pair potentials are parameterized by the cubic spline expansion, 


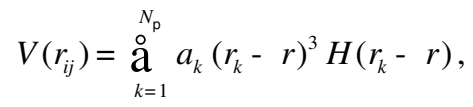

where $N_{\mathrm{p}}$ denotes the number of knots, $a_{k}$ are the fitting parameters and $H$ denotes the Heaviside unit step function.

\section{Interatomic potential fitting strategy}

Formatted: Bullets and Numbering

For the study of radiation damage production and evolution, a potential must reproduce the alloy's thermodynamics, kinetics and defect interactions reasonably well. The kinetics of the alloy transformation is largely defined by point-defect migration barriers for particular configurations of solute atoms around the defect in the host matrix. The defect interactions are characterized by the binding energies between solute complexes and solute point-defect complexes.

The Fe potential used in this work has been widely used, tested and has demonstrated so far to be the one that succeeds at best in reproducing the properties of iron that are of interest for radiation damage studies [32], including dislocation properties [33]. The $\mathrm{Cu}$ and $\mathrm{Ni}$ potentials, though not specifically developed to model radiation damage, represent state-ofthe-art potentials that are suitable for our purposes. The $\mathrm{Fe}-\mathrm{Cu}$ and $\mathrm{Fe}-\mathrm{Ni}$ interactions were fitted using the experimental phase diagram as direct reference, while using DFT indications to fit the point-defect/solute-atom interaction and vacancy migration barriers, as described in [30, 31, 34].

The $\mathrm{Cu}-\mathrm{Ni}$ interaction is here fitted to a Calphad parameterized mixing enthalpy [35]. Since the $\mathrm{Cu}-\mathrm{Ni}$ system consists of a simple miscibility gap of two fcc phases [35-38], a fit to the randomly disordered mixing enthalpy proved sufficient for a reasonable reproduction of the Calphad parameterized miscibility gap [34]. Information about point defects was included by fitting to the interaction energy of $\mathrm{Ni}-\mathrm{Cu}$ pairs and the $\mathrm{Ni}-\mathrm{Cu}\langle 110\rangle$ dumbbell, all obtained via DFT calculations in the bcc Fe matrix [21-22]. Note that no defect properties were fitted in the fcc Ni-Cu binary alloy and their proper description can therefore a priori not be guaranteed. Taking into account the above described thermodynamic properties for the $\mathrm{Cu}-\mathrm{Ni}$ binary and defect interactions in the Fe-Cu-Ni ternary, the spline coefficients in Eq. (5) are optimized. The parameters for all cross potentials are given in Appendix A. 
The thermal annealing of binary $\mathrm{Fe}-\mathrm{Cu}$ and ternary $\mathrm{Fe}-\mathrm{Cu}-\mathrm{Ni}$ alloys was modelled using a rigid lattice AKMC technique [39]. Initially, the $\mathrm{Cu}$ and $\mathrm{Ni}$ atoms are randomly distributed in a bcc Fe matrix. The evolution of the system leading to the atomic redistribution is driven by single vacancy diffusion. The diffusion jump frequency of the vacancy, $\Gamma$, is evaluated as a thermally activated process,

$$
\mathrm{G}=n_{0} \exp \left(-E_{\mathrm{m}} / k_{\mathrm{B}} T\right),
$$

where $v_{0}$ is an attempt frequency chosen to be constant and equal to $6 \times 10^{12} \mathrm{~s}^{-1}, E_{\mathrm{m}}$ is the local atomic environment (LAE)-dependent migration energy, $k_{\mathrm{B}}$ is Boltzmann's constant and $T$ is the absolute temperature. Note that a priori the attempt frequency $v_{0}$ is both temperature and LAE-dependent through magnetic and vibrational entropy; however, variations of $\Gamma$ due to the LAE-dependence of $E_{\mathrm{m}}$ are numerically of significantly more importance [40]. Thus, a proper estimation of $E_{\mathrm{m}}$ is essential for a correct description of the micro-chemical evolution of the alloy.

In the literature, many approximations to describe $E_{\mathrm{m}}$ in terms of the LAE exist; for example, the Kang-Weinberg decomposition [41] based on total energy differences and broken-bond counting [42]. However, as shown in [40, 43], $E_{\mathrm{m}}$ estimated in such a way may be very approximate as compared to a proper barrier estimation, for example by using drag [44] or nudged elastic band (NEB) methods [45]. Recently, a regression method based on artificial neural networks (ANNs) was developed that closely reproduces NEB obtained values of $E_{\mathrm{m}}$ at the same computational cost as the Kang-Weinberg decomposition [43, 46, 47]. In this work, the latter method was chosen and applied to compute $E_{\mathrm{m}}$ during the AKMC simulations.

In short, the method works as follows. First, an adequate amount $\left(\sim 10^{5}\right)$ of NEBobtained migration barriers, calculated with our potential, is produced, for randomly chosen LAE configurations. The obtained values inherently include $0 \mathrm{~K}$ relaxation effects. Thus, a database of migration barrier examples is built: $40 \%$ is used for the training of the ANN, while $\sim 60 \%$ is used for the validation of the ANN predicted values. This strategy provided an ANN optimised to predict $E_{\mathrm{m}}$ for LAEs encountered during simulated thermal annealing. More details on the applied method are provided in [43, 46, 47]. 
In a bcc lattice, there are eight possible first nearest neighbour sites whereto a single vacancy can jump (exchanges with farther neighbours require much higher migration energies). In the model adopted here, each vacancy jump corresponds to a Monte Carlo step. The jump to be performed is chosen based on its probability, evaluated in terms of jump frequency. The time between two jumps is calculated according to a mean residence time algorithm, i.e. the inverse of the sum of the eight possible jump frequencies [39].

Finally, for the purpose of validating our potential, we performed AKMC simulations on the $\mathrm{Fe}-1.13 \mathrm{Cu}$ and $\mathrm{Fe}-1.13 \mathrm{Cu}-1.36 \mathrm{Ni}$ alloys at $823 \mathrm{~K}$, for which experimental data are available in the literature [48]. The AKMC simulations were performed in cubic boxes containing 128,000 atoms until coarsening regime was reached, which amounts to a MC time, $t_{\mathrm{MC}}$, of $\sim 0.3 \mathrm{~s}$. As further discussed in Section 4 , the MC time in a thermal ageing simulation cannot be directly compared to real time: the corresponding real time is much longer and to estimate it an appropriate way time renormalization must be applied [49].

\section{Results}

\section{Static properties}

Formatted: Bullets and Numbering

The binding energies (positive sign indicating attractive interaction) of the ternary defect complexes, calculated by molecular statics (MS) with our potential, are compared with the corresponding DFT values [21-22] in Table 1. The latter data suggest a negligible interaction between isolated $\mathrm{Cu}$ and $\mathrm{Ni}$ atoms in the bcc Fe matrix and the instability of the mixed $\langle 110\rangle$ dumbbell, for both solutes. Both effects are reasonably well reproduced by our potential and suggest a reasonable defect behaviour description.

Regarding the thermodynamic behaviour of our potential, the mixing enthalpy obtained by MS in random alloys is compared to the most recent ones from the Calphad database [35, 38], in Fig. 1. Clearly, the agreement with the parameterization by Srikanth et al. (the target curve) is very good. The miscibility gap calculated with our potential is compared with the two existing ones built from the Calphad database $[35,38]$ in Fig. 2. The miscibility gap based on our potential was calculated using MC simulations in the semi-grand canonical (transmutation) ensemble, as explained in more detail in [34]. Given the limited degree of freedom for fitting (due to the already predetermined electronic density weights $\rho_{\mathrm{Fe}} / \rho_{\mathrm{Cu}}$ and $\left.\rho_{\mathrm{Fe}} / \rho_{\mathrm{Ni}}\right)$, and the large variation in the miscibility gap built from the Calphad database, the agreement between potential and database can be considered reasonable. 
Prior to the simulated thermal annealing, we performed static calculations to study the stability range of coherent $\mathrm{Cu}$ precipitates in the bcc Fe-matrix and its interaction with $\mathrm{Ni}$. Firstly, pure coherent $\mathrm{Cu}$ precipitates of different sizes were relaxed by means of MS calculations. During the relaxation, the coordination number of the atoms inside the $\mathrm{Cu}$ precipitate was monitored to reveal possible crystallographic transformations. From these calculations it was found that $\mathrm{Cu}$ precipitates with a size up to $2.5 \mathrm{~nm}$ remain stable. Above this size, the precipitate tries to change its bulk crystal structure. Experimentally, $\mathrm{Cu}$ precipitates were observed to be coherent with the bcc Fe-matrix up to an average size of 4-5 $\mathrm{nm}$, after which they transform into a $9 \mathrm{R}$ and eventually into a fcc structure [50-52]. We thus conclude that the stability range of coherent bcc $\mathrm{Cu}$ precipitates predicted by our potential is in the correct range, though perhaps somewhat underestimated.

Secondly, we performed MS calculations to examine the interaction between a $\mathrm{Cu}$ precipitate and a $\mathrm{Ni}$ atom in the bcc Fe-matrix. To do so, the binding energy between a Ni atom and a $\mathrm{Cu}$ precipitate (of size 1,2 and $2.5 \mathrm{~nm}$ ) was calculated as a function of the distance between the $\mathrm{Ni}$ atom and centre of the $\mathrm{Cu}$ precipitate. To increase statistics, the $\mathrm{Ni}$ atom was moved along different crystallographic directions $(\langle 100\rangle,\langle 110\rangle,\langle 111\rangle$ and $\langle 112\rangle)$. The results of these calculations are presented in Fig. 3, where the abscissa is the distance from the $\mathrm{Cu}$ precipitate centre in reduced units (with $R_{\mathrm{p}}$ the precipitate radius). The figure clearly shows strong attractive interaction between the $\mathrm{Ni}$ atom and the surface of the $\mathrm{Cu}$ precipitate. This attractive interaction decreases fast with increasing distance between atom and precipitate. For a $\mathrm{Ni}$ atom inside the $\mathrm{Cu}$ precipitate, the interaction is always repulsive. The curves presented in Fig. 3 are in agreement with the experimental observations of Nienrichment at the surface of $\mathrm{Cu}$ precipitates $[3,4,48]$. In addition, it is important to note that the binding energy curves for the unrelaxed configurations are almost coincident with the binding energy curves for the relaxed configurations presented in Fig. 3. This observation suggests that the binding of $\mathrm{Ni}$ on a $\mathrm{Cu}$ precipitate surface is a chemical effect, rather than an effect due to the strain field around the (oversized) $\mathrm{Cu}$ precipitate. This finding partially justifies the application of a rigid lattice MC technique to simulate the thermal annealing of $\mathrm{Fe}-\mathrm{Cu}-\mathrm{Ni}$ alloys.

\section{Simulated thermal ageing}

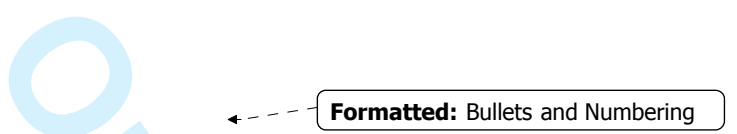

Visual inspection of the configurations obtained during the simulations show that for both studied alloys $\mathrm{Cu}$-rich precipitates are formed. The Ni distribution in the ternary alloy appears 
to remain random under the present thermal ageing conditions. From the analysis of the extracted configurations, the precipitate density, $N_{\mathrm{p}}$, mean diameter, $d_{\mathrm{p}}$, and solute concentration in the matrix, $x_{\mathrm{S}}$, were computed as a function of $t_{\mathrm{MC}}$. These results for both alloys are shown in Figs. 4, 5 and 6, respectively. From these figures, supported by visual inspection, the precipitation process appears to occur by the nucleation, growth and coarsening. The steep increase in $N_{\mathrm{p}}$ during the first fractions $(\sim 5 \mathrm{~ms})$ of the simulation denotes the nucleation process. At the maximum density, $N_{\mathrm{p}}$ remains approximately constant and $d_{\mathrm{p}}$ steadily increases. This, combined with a steep decrease of $x_{\mathrm{Cu}}$ in the matrix, indicates a stage of dominant growth in the interval $\sim 5-25 \mathrm{~ms}$. Beyond $25 \mathrm{~ms}, N_{\mathrm{p}}$ decreases with $t_{\mathrm{MC}}$ while $d_{\mathrm{p}}$ keeps increasing, which indicates the coarsening stage, where large precipitates grow at the cost of the dissolution or coalescence of smaller ones. However, the simultaneous decrease of $x_{\mathrm{Cu}}$, even beyond $25 \mathrm{~ms}$, indicates that the precipitation occurs by a mixture of growth and coarsening.

As already mentioned, the $\mathrm{Ni}$ atoms in the matrix remain randomly distributed during the whole simulation. At the nucleation stage, however, they act as nucleation centres for $\mathrm{Cu}$ precipitates. This clearly follows from Fig. 4, where the peak density for the Fe-Cu-Ni alloy is $\sim 29 \%$ higher than for the Fe-Cu alloy. This is in agreement with experimental observations by Buswell et al. [48], where a density increase of $\sim 34 \%$ was observed due to the addition of $\mathrm{Ni}$. This observation is further supported by the observation that $x_{\mathrm{Ni}}$ in the matrix decreases during the nucleation stage and then immediately saturates to its equilibrium value. Since $x_{\mathrm{Ni}}$ remains constant during growth and coarsening stage, $\mathrm{Ni}$ does not migrate from the matrix to the $\mathrm{Cu}$ precipitate surface. Instead, the $\mathrm{Ni}$ enclosed in the $\mathrm{Cu}$ precipitates due to nucleation and growth is expelled to the precipitate surface. This is also consistent with the static calculations presented in Section 3.1, and rigid lattice MC simulations in the Refs. [17, 18, 21]. In Fig. 5, we also observe that $d_{\mathrm{p}}$ is lower, due to the addition of $\mathrm{Ni}$, which is also in qualitative agreement with experimental observations by Buswell et al. [48]. Near the end of our simulation, however, both $N_{\mathrm{p}}$ and $d_{\mathrm{p}}$, with and without $\mathrm{Ni}$, converge to a common value, which is also observed in the latter experiment.

An example for a typical size distribution of the Cu-rich precipitates during the late growth/early coarsening is plotted in Fig. 7, which shows a multi-modal distribution of the precipitate size (each mode corresponds to a Cu-rich precipitate). With increasing $t_{\mathrm{MC}}$, the mean size of the clusters increases (as shown in Fig. 4) and the maximum density in the first mode decreases (due to the depletion of the matrix and dissolution of small clusters). However, the distribution of small clusters $(<10$ atoms) remains non-zero during the whole 
simulation. Past the nucleation stage, when no new clusters are nucleated, such small clusters are below the critical size for nucleation. Therefore, the maximum cluster size for which this distribution is non-zero gives an estimate for the critical cluster size for nucleation. Above this size, the nuclei are stable and spontaneously grow into $\mathrm{Cu}$-rich precipitates, which are part of the second mode of the distribution. Following this logic, we estimate the critical cluster size to be $\pm 5-8$ atoms, which is well below the resolution of any experimental technique.

\section{Discussion}

Prior to discussing the AKMC results, we discuss the quality of the migration barriers estimated by our ANN. The quality of the agreement between the ANN predictions and NEB calculated barriers defines how good the ANN represents our potential. In Fig. 8 the barriers calculated using NEB and our potential are compared to the ANN predicted ones for the Fe$\mathrm{V}, \mathrm{Cu}-\mathrm{V}$ and $\mathrm{Ni}-\mathrm{V}$ exchanges for randomly chosen LAEs, as described in Section 2.3. From the data in the figure we calculated that the average error for $\mathrm{Fe}-\mathrm{V}$ exchange is $3.7 \%$, for $\mathrm{Cu}$ $\mathrm{V}$ exchange it is $3.5 \%$ and for $\mathrm{Ni}-\mathrm{V}$ exchange it is $4.3 \%$. As a comparison, a more heuristic estimation of $E_{\mathrm{m}}$, for example by the Kang-Weinberg decomposition [40], provides average errors amounting to $52.0 \%$. Therefore we believe that, at present, the ANN provides the best compromise between accuracy and computation time to estimate the LAE dependent $E_{\mathrm{m}}$. A more detailed discussion on this subject can be found in Refs [43, 47].

In our AKMC simulations, the vacancy concentration is $1 / N$ and is imposed by the box size, $N$ being the number of atoms in the box. This concentration is typically a large overestimation of the physical equilibrium vacancy concentration in an alloy. To account for this, $t_{\mathrm{MC}}$ must be rescaled to obtain the real physical annealing time, $t_{\text {real }}$. Here, we use a method described in Refs. [41,53] and briefly describe the procedure below. During the simulations, the vacancy concentration in the matrix, $C_{\mathrm{V}}^{\mathrm{MC}}(\mathrm{M})$, defined as the concentration on sites without $\mathrm{Cu}$ atoms among their first and second nearest neighbours, is computed as,

$$
C_{\vee}^{\mathrm{MC}}(\mathrm{M})=\frac{f_{\mathrm{V}}^{\mathrm{M}}}{N X_{\mathrm{M}}}
$$


Here $f_{\mathrm{V}}^{\mathrm{M}}$ is the fraction of time spent by the vacancy in the matrix and $X_{\mathrm{M}}$ is the atomic fraction of the matrix main element $\left(X_{\mathrm{M}} \approx 1\right.$ in dilute alloys as those considered here). The true vacancy equilibrium concentration in the matrix material, $C_{\mathrm{V}}^{\mathrm{eq}}(\mathrm{M})$, is given as,

$$
C_{\mathrm{V}}^{\mathrm{eq}}(\mathrm{M})=\exp \left(-E_{\mathrm{f}}^{\mathrm{\vee}} / k_{B} T\right),
$$

$E_{\mathrm{f}}^{\vee}$ being the formation energy of a vacancy in the matrix, thereby neglecting the entropic contributions. As a result, $t_{\text {real }}$ is related to $t_{\mathrm{MC}}$ via the equation,

$$
t_{\text {real }}=t_{\mathrm{MC}} \frac{C_{\mathrm{V}}^{\mathrm{MC}}(\mathrm{M})}{C_{\mathrm{V}}^{\mathrm{eq}}(\mathrm{M})}
$$

This scheme of time renormalization guarantees a diffusion coefficient of the solutes in the matrix that remains constant during the precipitation process. Following this procedure, we find a conversion factor of $\sim 23$ for the Fe-Cu binary alloy, where we took $E_{\mathrm{f}}^{\vee}=1.71 \mathrm{eV}$ and $f_{\mathrm{V}}^{\mathrm{M}}=10^{-4}$ as calculated in our simulations. The maximum obtained $t_{\mathrm{MC}}$, on the other hand, is of the order of $1 \mathrm{~s}$; therefore the maximum $t_{\text {real }}$ obtained in our simulations is of the order of $23 \mathrm{~s}$. This time is at least an order of magnitude shorter than typical (short) experimental annealing times that are of the order of hours. Therefore, direct quantitative comparison with thermal ageing experiments is impossible and only a qualitative comparison was presented above.

The short physical annealing time is also the reason for small precipitate size, $d_{\mathrm{p}} \sim 1 \mathrm{~nm}$, and large precipitate density, $N_{\mathrm{p}} \sim 10^{25} \mathrm{~m}^{-3}$, obtained during our simulations. In experiments, typical values for precipitate size and density are $d_{\mathrm{p}} \sim 4 \mathrm{~nm}$ and $N_{\mathrm{p}} \sim 10^{23} \mathrm{~m}^{-3}$, respectively, for an ageing time of $2 \mathrm{~h}$ at the same temperature as our simulations [48]. Furthermore, the limited box size used for our simulations is also a restrictive factor. If we assume that all the $\mathrm{Cu}$ in our simulation box forms one spherical precipitate, its size would be $3.2 \mathrm{~nm}$ at most and the corresponding density would be $6.7 \times 10^{23} \mathrm{~m}^{-3}$, which gives an upper and lower limit for $d_{\mathrm{p}}$ and $N_{\mathrm{p}}$, respectively. For a proper comparison to experiments, we should thus also increase the simulation box by at least an order of magnitude. Noting that the physical simulation time shows a quadratic increase with box size [54], it is clear that our AKMC tool in its current implementation is not adequate for direct comparison with experiments. 
To achieve the latter, the AKMC method must be accelerated by a few orders of magnitude. Because in our simulations the vacancy is trapped inside the precipitate by more than $99 \%$ of the computation time $\left(f_{\mathrm{V}}^{\mathrm{M}}=10^{-4}\right)$, a lot of computation time is spent which does not contribute to precipitate growth. Currently, schemes where $f_{\mathrm{V}}^{\mathrm{M}}$ is significantly reduced by slightly adapting the AKMC algorithm while keeping the correct physical time evolution of the system are under investigation. Such a scheme in combination with parallelisation of the code would speed up our simulations by an order of magnitude. Other schemes that are under consideration, specifically in the pure coarsening stage, are first passage acceleration methods [55].

Despite the limitations of the AKMC simulations, good qualitative agreement with experiments was found. We stress the fact that the combination of a ternary many-body interatomic potential and the ANN based AKMC is able to produce very accurate time dependent simulations. Due to the many-body character of our potential, the results are directly transferrable to MD or other schemes that can account for relaxations. This is impossible to accomplish for the case of simple pair bonding potentials or DFT parameterizations used in other works $[21,22,41,49,53]$.

\section{Summary and conclusions}

A many-body interatomic potential for the ternary $\mathrm{Fe}-\mathrm{Cu}-\mathrm{Ni}$ alloy was developed for the purpose of modelling radiation damage in reactor pressure vessel steels. For this purpose, we used previously developed, state-of-the-art $\mathrm{Fe}-\mathrm{Cu}$ and $\mathrm{Fe}-\mathrm{Ni}$ potentials, expressedly aimed at modelling radiation damage. The $\mathrm{Cu}-\mathrm{Ni}$ cross potential was fitted to the experimental phase diagram and the DFT calculated interaction energies of $\mathrm{Cu}-\mathrm{Ni}$ defect complexes in the bcc $\mathrm{Fe}$ matrix, so that a truly ternary potential was obtained.

The potential was validated by computing the $\mathrm{Cu}-\mathrm{Ni}$ phase diagram, the interactions between $\mathrm{Cu}$ precipitates and $\mathrm{Ni}$, and a simulated thermal annealing of the $\mathrm{Fe}-1.13 \mathrm{Cu}$ and $\mathrm{Fe}$ $1.13 \mathrm{Cu}-1.36 \mathrm{Ni}$ alloys at $823 \mathrm{~K}$. The obtained phase diagram was found to be in reasonable agreement with the experimental one. The static calculations revealed a strong attraction between $\mathrm{Ni}$ and a $\mathrm{Cu}$ precipitate surface, which is consistent with experimental observations. In the thermal annealing simulations good qualitative agreement with experiments was established. The effects of the addition of $\mathrm{Ni}$ on average precipitate size and density showed similar trends as observed in experiments, notably increased density and reduced size, as a consequence of $\mathrm{Ni}$ enhancing $\mathrm{Cu}$ precipitate nucleation. In agreement with atom-probe 
studies, $\mathrm{Cu}$ precipitates were found to be enriched by $\mathrm{Ni}$ on the precipitate surface. The latter effect was determined to be the consequence of the explusion of $\mathrm{Ni}$ from inside fully grown precipitates, rather than of transport of $\mathrm{Ni}$ atoms from the matrix to the surface of the precipitate. Quantitative comparison with experiments, however, could not be established, due to the inherent limitations of the used simulation techniques.

To conclude, we emphasize that, to the best of our knowledge, our Fe-Cu-Ni potential represents the first example of a ternary many-body potential developed to study radiation damage. Due to the many-body character of the potential, results obtained by different atomistic techniques are easily transferable, in contrast to the models currently available in the literature.

\section{Acknowledgements}

This work was performed in the framework of the FP6/PERFECT project, partially supported by the European Commission (EC), under contract FI60-CT-2003-5088-40. It also contributes to the EC funded FP7/PERFORM60 project, grant agreement 232612. The work was also sponsored by the SECYT-FWO bilateral cooperation agreement, Project FW/07/EXII/002. RCP wishes to acknowledge partial support from CONICET-PIP 5062.

\section{Appendix A: Parameterization of the cross potentials}

The knots and fitting coefficients used in the cubic spline expansion (3) describing the mixed pair interactions are given in Table $\mathrm{Al}$ for the $\mathrm{Fe}-\mathrm{Cu}, \mathrm{Fe}-\mathrm{Ni}$ and $\mathrm{Cu}-\mathrm{Ni}$ systems. The potentials used to describe the pure species, $\mathrm{Fe}, \mathrm{Cu}, \mathrm{Ni}$, are given in the works by Mendelev et al. [25], Mishin et al. [26] and Voter and Chen [27], respectively. As mentioned in Section 2 all pure potentials must be transformed to the effective potential gauge to be compatible with the cross potentials derived here. With the superscript 'eff' denoting the pure potential functions, the needed transformation $\hat{T}$ is,

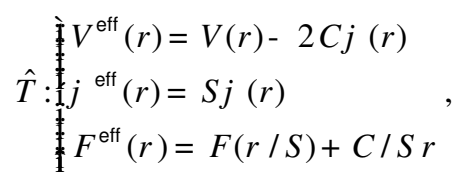


where $C$ and $S$ are constants given in Table A2. Tabulations of all the binary potentials can be found on-line following the link: http://www.ctcms.nist.gov/potentials/.

\section{References}

[1] J. P. Massoud, S. Bugat, J. L. Boutard, D. Lidbury, S. Van Dyck, F. Sevini, "Prediction of irradiation damage effects on reactor components (PERFECT)", in: "FISA 2006 EU Research and Training in Reactor Systems”, G. Van Goethem, P. Manolatos, M. Hugon, V. Bhatnagar, S. Casalta and M. Deffrennes Eds., Directorare-General for Research, Euratom (Luxembourg, 2006).

[2] P. Pareige, J.C. Van Duysen and P. Auger, Appl. Surf. Sci. 67 (1993) p. 342.

[3] P. Pareige and M.K. Miller, Appl. Surf. Sci. 94/95 (1996) p. 370.

[4] M.K. Miller and K.F. Russell, J. Nucl. Mater. 371 (2007) p. 145.

[5] J. Kameda and A.J. Bevolo, Acta Metall. 37 (1989) p. 3283.

[6] Y. Nishiyama, K. Onizawa and M. Suzuki, J. ASTM Intl. 4 (2007) p. 83.

[7] U. Potapovs and J.R. Hawthorne, Nucl. Appl. 1 (1969) p. 27.

[8] G. Odette, Scr. Metall. 17 (1983) p. 1183.

[9] W. Phythian, C. English, J. Nucl. Mater. 205 (1993) p. 162.

[10] P. Auger, P. Pareige, A. Akamatsu, D. Blavette, J. Nucl. Mater. 225 (1995) p. 225.

[11] J.T. Buswell, W.J. Phytian, R.J. McElroy, S. Dumbill, P.H.N. Ray, J. Mace and R.N. Sinclair, J. Nucl. Mater. 225 (1995) p. 196.

[12] C. English, W. Phythian, R. McElroy, Mater. Res. Soc. Symp. Proc., vol. 439, MRS, Pittsburg, Pennsylvania, 1997, p. 471.

[13] G.R. Odette and G.E. Lucas, JOM 53 (2001) p. 18.

[14] Y. Nagai, Z. Tang, M. Hassegawa, T. Kanai, M. Saneyasu, Phys. Rev. B 63 (2001) p. 134110 .

[15] K. Morita, S. Ishino, T. Tobita, Y. Chimi, N. Ishikawa, A. Iwase, J. Nucl. Mater. 304 (2002) p. 153.

[16] R. Chaouadi, R. Gérard, J. Nucl. Mater. 345 (2005) p. 65.

[17] C.L. Liu, G.R. Odette, B.D. Wirth and G.E. Lucas, Mater. Sci. Eng. A 238 (1997) p. 202.

[18] G.R. Odette and B.D. Wirth, J. Nucl. Mater. 251 (1997) p. 157.

[19] M.W. Finnis and J.E. Sinclair, Phil. Mag. A 50 (1984) p. 45.

[20] S.M. Foiles, M.I. Baskes and M.S. Daw, Phys. Rev. B 33 (1986) p. 7983.

[21] E. Vincent, C.S. Becquart and C. Domain, J. Nucl. Mater. 351 (2006) p. 88. 
[22] E. Vincent, C.S. Becquart and C. Domain, Nucl. Instr. and Meth. B 255 (2007) p. 78.

[23] "Predicting Radiation-Induced Transition Temperature Shift in Reactor Vessel Materials, E706 (IIF)”, ASTM International, E 900 - 02, 2007.

[24] M.S. Daw and M.I. Baskes, Phys. Rev. B 29 (1984) p. 6443.

[25] M.I. Mendelev, A. Han, D.J. Srolovitz, G.J. Ackland, D.Y. Sun and M. Asta, Phil. Mag. A 83 (2003) p. 3977.

[26] Y. Mishin, M.J. Mehl, D.A. Papaconstantopoulos, A.F. Voter, J.D. Kress, Phys. Rev. B 63 (2001) p. 224106.

[27] A.F. Voter and S.P. Chen, Mater. Res. Soc. Symp. Proc. 82 (1987) p. 175.

[28] F. Ercolessi, M. Parrinello and E. Tosatti, Phil. Mag. A 58 (1988) p. 213.

[29] R.A. Johnson and D.J. Oh, J. Mater. Res. 4 (1989) p. 1195.

[30] R.C. Pasianot and L. Malerba, J. Nucl. Mater. 360 (2007) p. 118.

[31] G. Bonny, R.C. Pasianot and L. Malerba, Model. Simul. Mater. Sci. Eng. 17 (2009) p. 025010 .

[32] L. Malerba, M.-C. Marinica, N. Anento, C. Björkas, H. Nguyen, C. Domain, F. Djurabekova, P. Olsson, K. Nordlund, A. Serra, D. Terentyev, F. Willaime and C.S. Becquart, "Comparison of empirical interatomic potentials for iron applied to radiation damage studies", submitted to J. Nucl. Mater. (2009).

[33] C. Domain and G. Monnet, Phys. Rev. Lett. 95 (2005) p. 215506.

[34] G. Bonny, R.C. Pasianot and L. Malerba, "Fitting interatomic potentials consistent with thermodynamics: $\mathrm{Fe}, \mathrm{Cu}, \mathrm{Ni}$ and their alloys", submitted to Philos. Mag. (2009).

[35] S. Srikanth and K.T. Jacob, Mater. Sci. Technol. 5 (1989) p. 427.

[36] R.C. Sharma, Trans. Indian Inst. Met. 35 (1982) p. 372.

[37] S. Mey, Z. Metallkde. 78 (1987) p. 502.

[38] S. Mey, CALPHAD 16 (1992) p. 255.

[39] W.M. Young and E. W. Elcock, Proc. Phys. Soc. London 89 (1966) p. 735.

[40] F.G. Djurabekova, R. Domingos, G. Cerchiara, N. Castin, E. Vincent and L. Malerba, Nucl. Instr. and Meth. B 255 (2007) p. 8.

[41] H.C. Kang and W.H. Weinberg, J. Chem. Phys. 90 (1989) p. 2824.

[42] Y. Le Bouar and F. Soisson, Phys. Rev. B 65 (2002) p. 094103.

[43] N. Castin and L. Malerba, "Prediction of point defect migration energy barriers in alloys using artificial intelligence for atomistic kinetic Monte Carlo applications", accepted in Nucl. Instr. and Meth. (2009). 
[44] G. Henkelman, G. Jóhannesson and H. Jónsson, "Methods for Finding Saddle Points and Minimum Energy Paths”, Progress on Theoretical Chemistry and Physic, Ed. S.D. Schwartz (Kluwer Academic Publishers, 2000), p. 269.

[45] H. Jonsson, G. Mills, and K. W. Jacobsen, in Nudged Elastic Band Method for Finding Minimum Energy Paths of Transitions, Classical and Quantum Dynamics in Condensed Phase Simulations, Eds. B. J. Berne, G. Ciccotti, and D. F. Coker (World Scientific, Singapore, 1998).

[46] N. Castin, R.P. Domingos and L. Malerba, Intl. J. Comp. Intell. Sys. 1 (2008) p. 340.

[47] N. Castin and L. Malerba, "Artificial Intelligence regression of the vacancy migration energy to introduce relaxation effects in rigid lattice atomistic kinetic Monte Carlo simulations", submitted to J. Comp. Chem. (2009).

[48] J.T. Buswell, C.A. English, M.G. Hetherington, W.J. Phythian, G.D.W. Smith and G.M. Worrall, "An analysis of Small Clusters Formed in Thermally Aged and Irradiated $\mathrm{FeCu}$ and FeCuNi Model Alloys", Eds N.H. Packan et al., Effects of Radiation on Materials: 14th International Symposium (Volume II), ASTM STP 1046, Philadelphia, 1990, p. 127.

[49] E. Vincent, C.S. Becquart, C.Pareige, P. Pareige, C. Domain, J. Nucl. Mater. 373 (2008) 387.

[50] S. Pizzini, K.J. Roberts and W.J. Phythian, Phil. Mag. Lett. 61 (1990) p. 223.

[51] P.J. Othen, M.L. Jenkins, G.D.W. Smith and W.J. Phythian, Phil. Mag. Lett. 64 (1991) p. 383.

[52] F. Maury, N. Lorenzelli, M.H. Mathon, C.H. de Novion and P. Lagarde, J. Phys.: Condens. Matter. 6 (1994) p. 569.

[53] F. Soisson and C.-C. Fu, Phys. Rev. B 76 (2007) p. 214102.

[54] A. Chatterjee and D.G. Vlachos, J. Comput.-Aided Mater. Des. 14 (2007) p. 253.

[55] T. Opplestrup, V.V. Bulatov, G.H. Gilmer, M.H. Kalos and B. Sadigh, Phys. Rev. Lett. 97 (2006) p. 230602.

\section{Figure and table captions}

Figure 1 - Comparison between mixing enthalpy obtained from our potential with Calphad parameterized ones based on experiments $[35,38]$ for the $\mathrm{Cu}-\mathrm{Ni}$ binary.

Figure 2 - Comparison of the phase diagrams based on our potential and different Calphad parameterisations. 
Figure 3 - The binding energy between a $\mathrm{Ni}$-atom and $\mathrm{Cu}$ precipitate as a function of distance (reduced units).

Figure 4 - The mean precipitate density as a function of $t_{\mathrm{MC}}$.

Figure 5 - The mean precipitate diameter as a function of $t_{\mathrm{MC}}$.

Figure $6-$ The solute concentration in the matrix as a function of $t_{\mathrm{MC}}$.

Figure 7 - Typical size distribution for the Fe-1.13at.\% $\mathrm{Cu}$ at $823 \mathrm{~K}$ near the end of our simulations, averaged over 50 frames.

Figure 8 - Comparison of migration barriers obtained by NEB calculations and ANN predictions.

Table 1 - Defect binding energies in the bcc Fe matrix $(\mathrm{eV})$.

Table A1 - The fit parameters in Eq. (3) defining the cross pair interactions.

Table A2 - Transformation constants for the pure potentials.

\section{Figures and Tables}

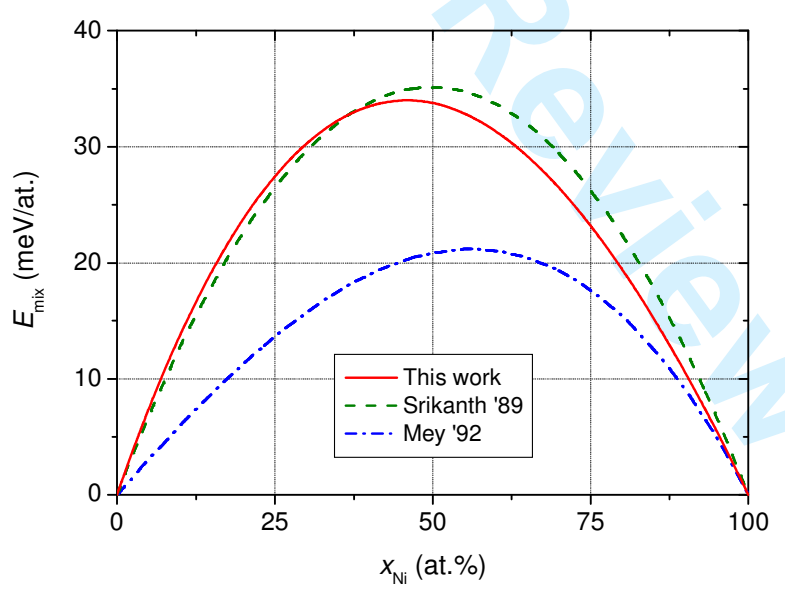

Fig. 1 


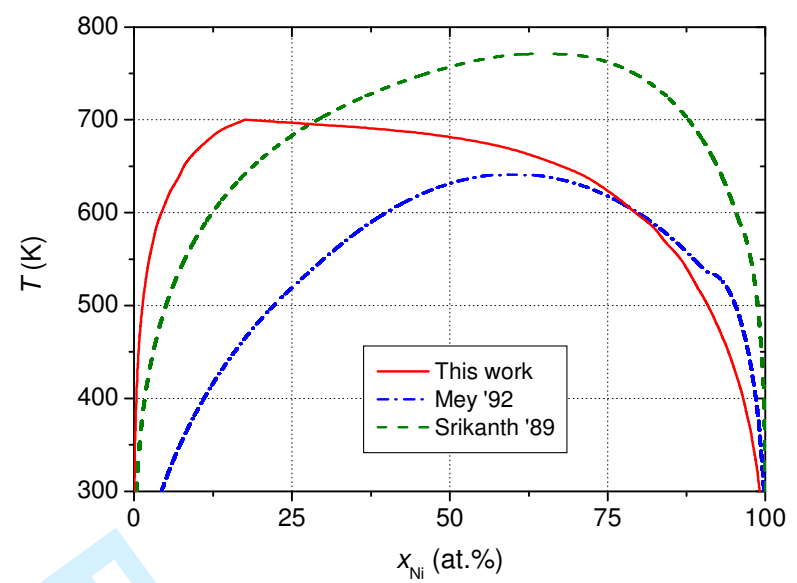

Fig. 2

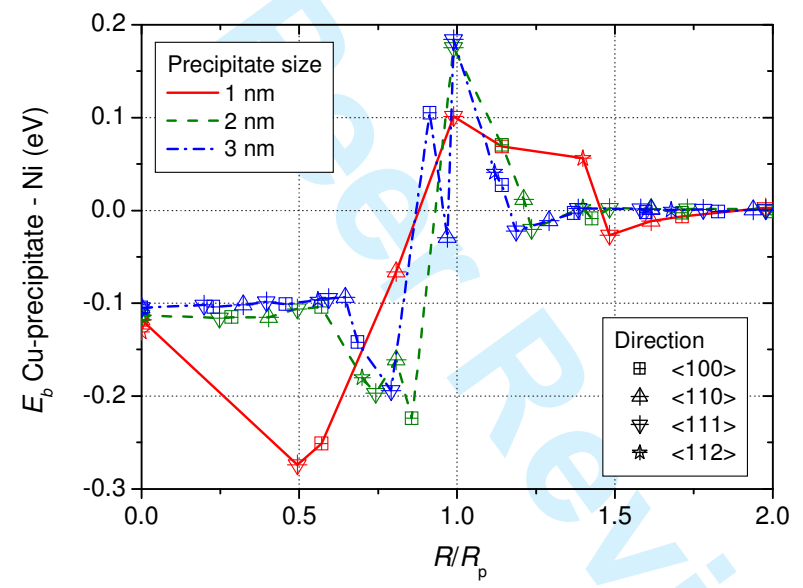

Fig. 3 


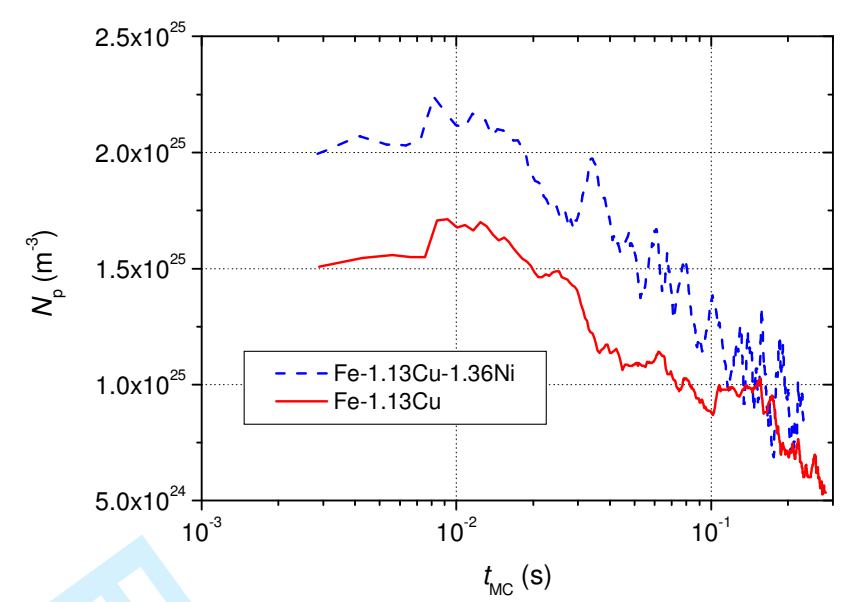

Fig. 4

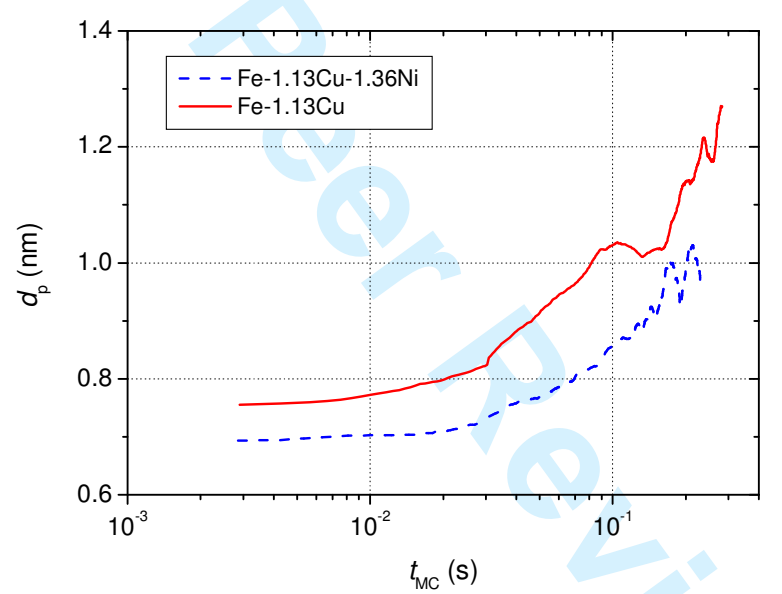

Fig. 5 


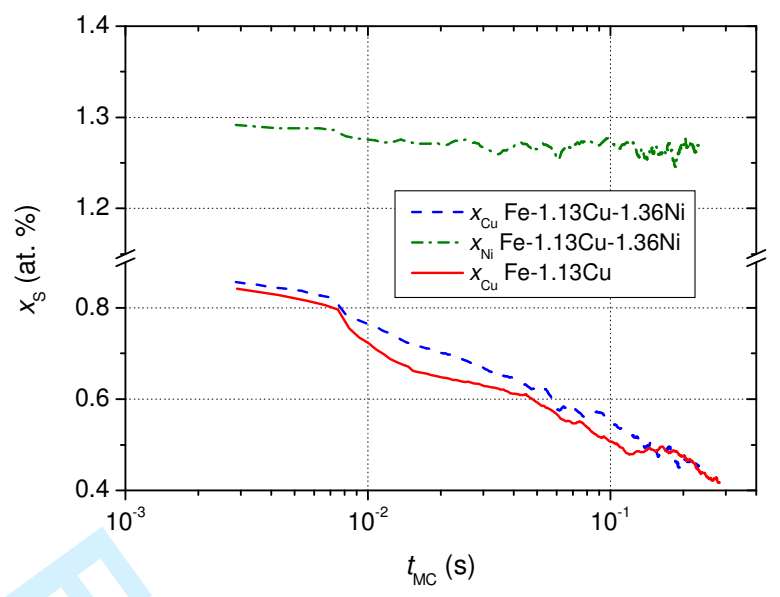

Fig. 6

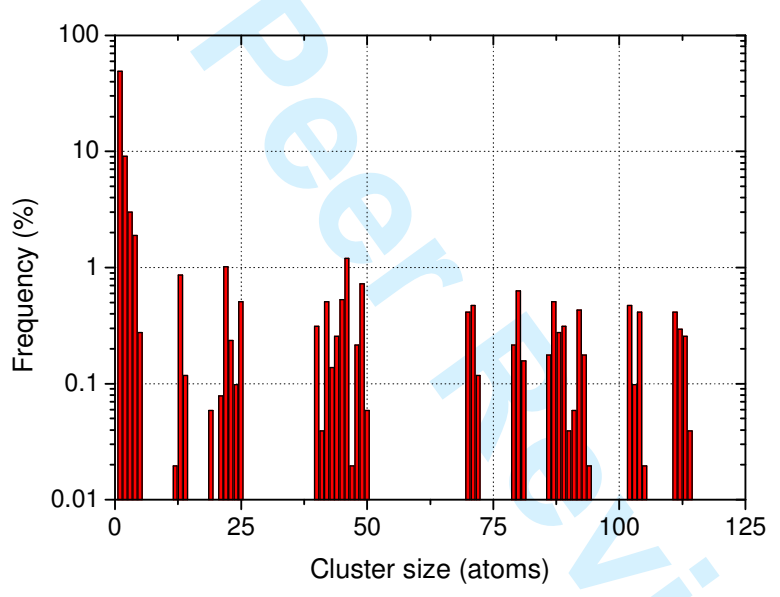

Fig. 7 
1

2

3

4

5

7

8

9

10

11

12

13

14

15

16

17

18

19

20

21

22

23

24

25

26

27

28

29

30

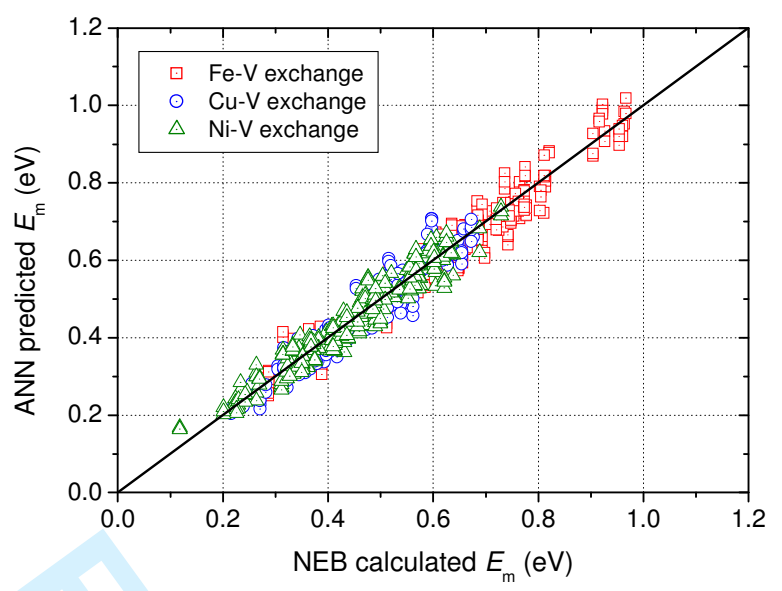

Fig. 8

Table 1

\begin{tabular}{lcc}
\hline & This Work & DFT \\
\hline$E_{\mathrm{b}} \mathrm{Cu}-\mathrm{Ni}(1 \mathrm{nn})$ & 0.01 & 0.02 \\
$E_{\mathrm{b}} \mathrm{Cu}-\mathrm{Ni}(2 \mathrm{nn})$ & 0.07 & -0.01 \\
$E_{\mathrm{b}}\langle 110\rangle \mathrm{Cu}-\mathrm{Ni}$ & -0.37 & -0.38 \\
\hline
\end{tabular}

Table A1

\begin{tabular}{|c|c|c|c|c|c|c|}
\hline \multirow[b]{2}{*}{$i$} & \multicolumn{2}{|c|}{$V_{\mathrm{Fe}-\mathrm{Cu}}$} & \multicolumn{2}{|c|}{$V_{\mathrm{Fe}-\mathrm{Ni}}$} & \multicolumn{2}{|c|}{$V_{\mathrm{Cu}-\mathrm{Ni}}$} \\
\hline & $r_{i}(\AA)$ & $a_{i}\left(\mathrm{eV} / \AA^{3}\right)$ & $r_{i}(\AA)$ & $a_{i}\left(\mathrm{eV} / \AA^{3}\right)$ & $r_{i}(\AA)$ & $a_{i}\left(\mathrm{eV} / \AA^{3}\right)$ \\
\hline 1 & 5.500000000 & $1.460151448 \mathrm{E}-03$ & 5.500000000 & $1.01819400 \mathrm{E}-02$ & 5.50000000 & $7.15210346 \mathrm{E}-03$ \\
\hline 2 & 5.257692308 & $-4.957562851 \mathrm{E}-02$ & 5.000000000 & $-1.24535646 \mathrm{E}-01$ & 5.18181818 & $-4.09422680 \mathrm{E}-02$ \\
\hline 3 & 5.015384615 & $6.998305005 \mathrm{E}-02$ & 4.500000000 & 4.08902943E-01 & 4.86363636 & $2.69045453 \mathrm{E}-02$ \\
\hline 4 & 4.773076923 & $-3.874280553 \mathrm{E}-03$ & 4.000000000 & $-7.37820671 \mathrm{E}-01$ & 4.54545455 & $1.25068302 \mathrm{E}-02$ \\
\hline 5 & 4.530769231 & $-8.723411300 \mathrm{E}-03$ & 3.500000000 & $5.91172697 \mathrm{E}-01$ & 4.22727273 & $-2.23538031 \mathrm{E}-02$ \\
\hline 6 & 4.288461538 & 6.496879404E-02 & 3.000000000 & 4.84506404E-01 & 3.90909091 & $-4.05296894 \mathrm{E}-04$ \\
\hline 7 & 4.046153846 & $3.695651396 \mathrm{E}-03$ & 2.500000000 & $1.24541131 \mathrm{E}+01$ & 3.59090909 & $-2.13007867 \mathrm{E}-02$ \\
\hline 8 & 3.803846154 & $-5.082318050 \mathrm{E}-01$ & 2.350000000 & $-2.50000000 \mathrm{E}+01$ & 3.27272727 & $8.15583164 \mathrm{E}-02$ \\
\hline 9 & 3.561538462 & $-3.076315854 \mathrm{E}-01$ & 2.200000000 & $5.00000000 \mathrm{E}+01$ & 2.95454545 & 3.73700354E-01 \\
\hline 10 & 3.319230769 & $2.117344729 \mathrm{E}+00$ & & & 2.63636364 & $1.92883049 \mathrm{E}+00$ \\
\hline 11 & 3.076923077 & $-1.203932506 \mathrm{E}+00$ & & & 2.31818182 & $8.25151398 \mathrm{E}+00$ \\
\hline 12 & 2.834615385 & 8.046491939E-03 & & & 2.30000000 & $-1.00000000 \mathrm{E}+01$ \\
\hline 13 & 2.592307692 & $2.703349799 \mathrm{E}+00$ & & & 1.90000000 & $1.00000000 \mathrm{E}+02$ \\
\hline 14 & 2.350000000 & $4.000000000 \mathrm{E}+01$ & & & & \\
\hline
\end{tabular}


Table A2

\begin{tabular}{ccc}
\hline Species & $C$ & $S$ \\
\hline $\mathrm{Fe}$ & 0.116093429 & 0.0380008812 \\
$\mathrm{Cu}$ & -0.00195739344 & 0.998555148 \\
$\mathrm{Ni}$ & -24.3575774 & 2.92527845 \\
\hline
\end{tabular}




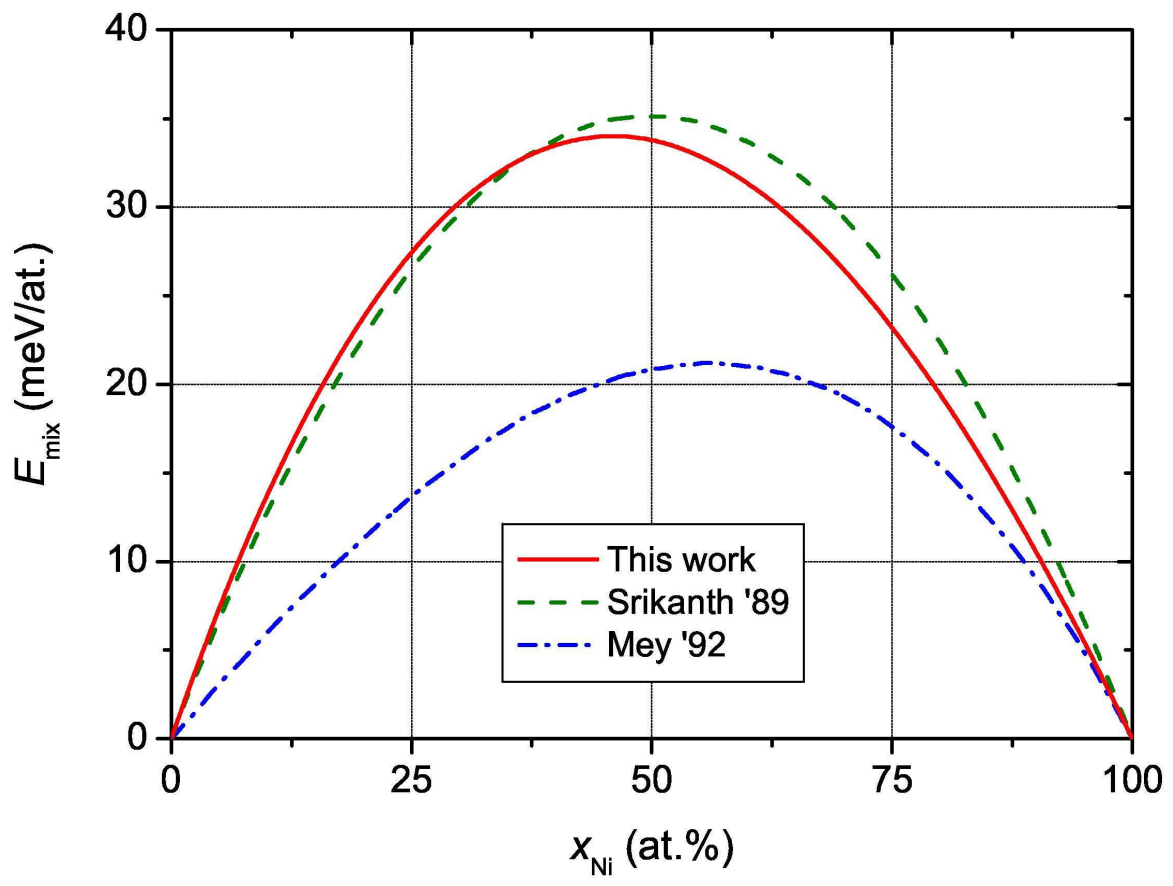

$106 \times 83 \mathrm{~mm}(600 \times 600 \mathrm{DPI})$ 


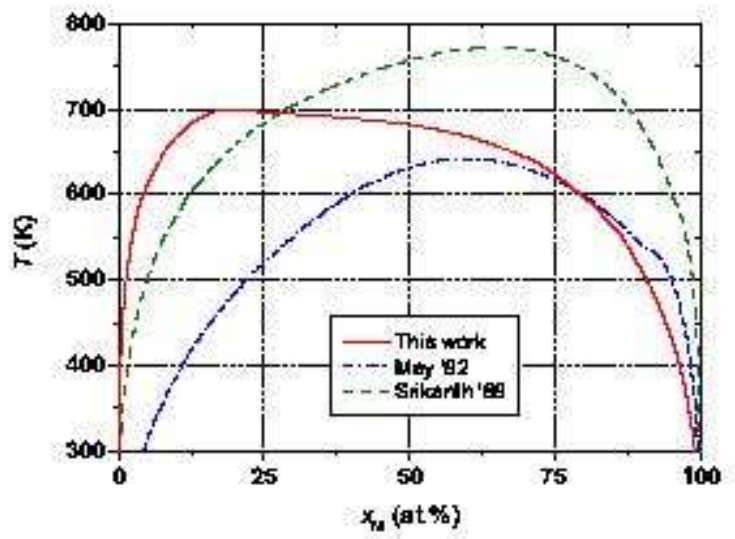

$107 \times 83 \mathrm{~mm}(72 \times 72 \mathrm{DPI})$

http://mc.manuscriptcentral.com/pm-pml 


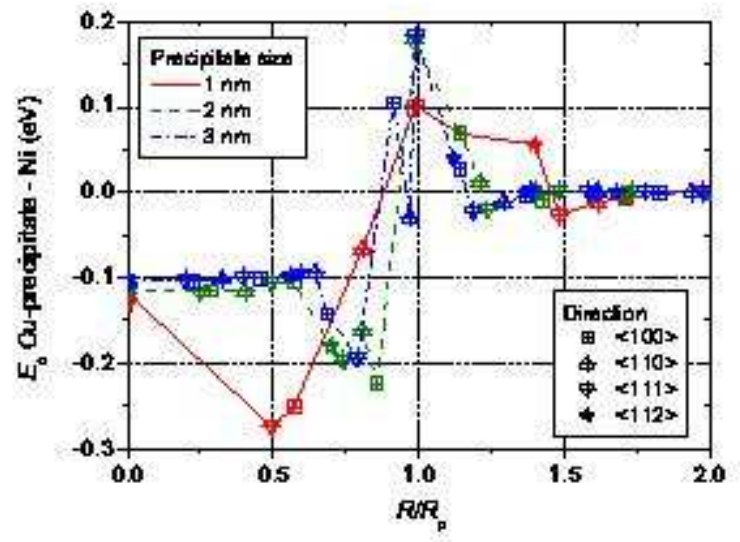

$108 \times 83 \mathrm{~mm}(72 \times 72 \mathrm{DPI})$ 


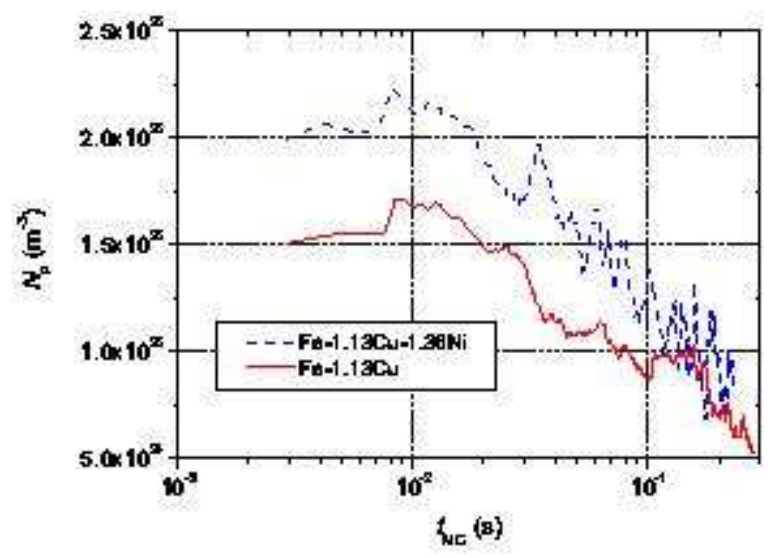

$112 \times 84 \mathrm{~mm}(72 \times 72 \mathrm{DPI})$

http://mc.manuscriptcentral.com/pm-pml 


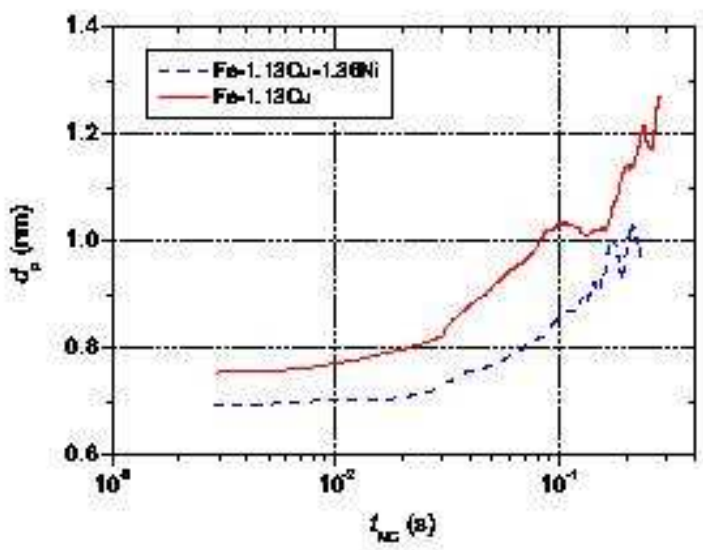

$104 \times 83 \mathrm{~mm}(72 \times 72$ DPI $)$ 


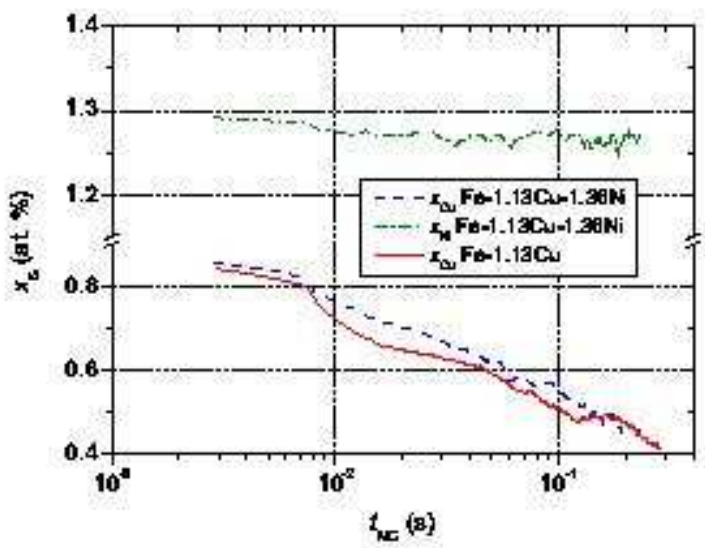

$105 \times 83 \mathrm{~mm}(72 \times 72 \mathrm{DPI})$

http://mc.manuscriptcentral.com/pm-pml 


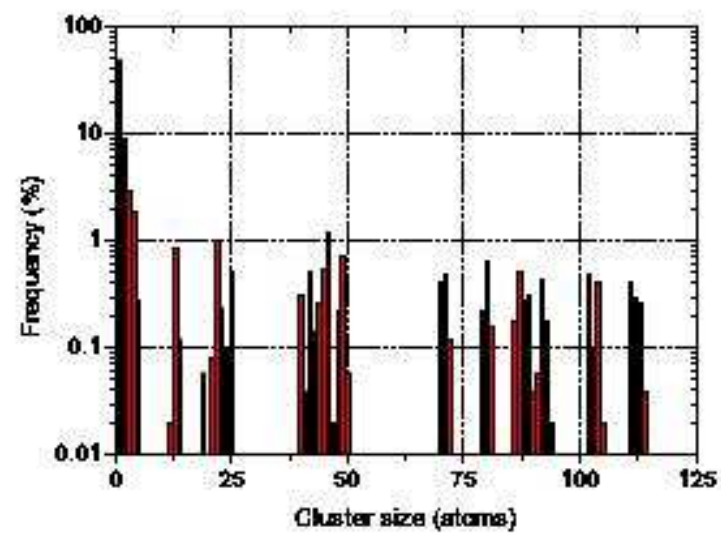

$106 \times 82 \mathrm{~mm}(72 \times 72 \mathrm{DPI})$ 


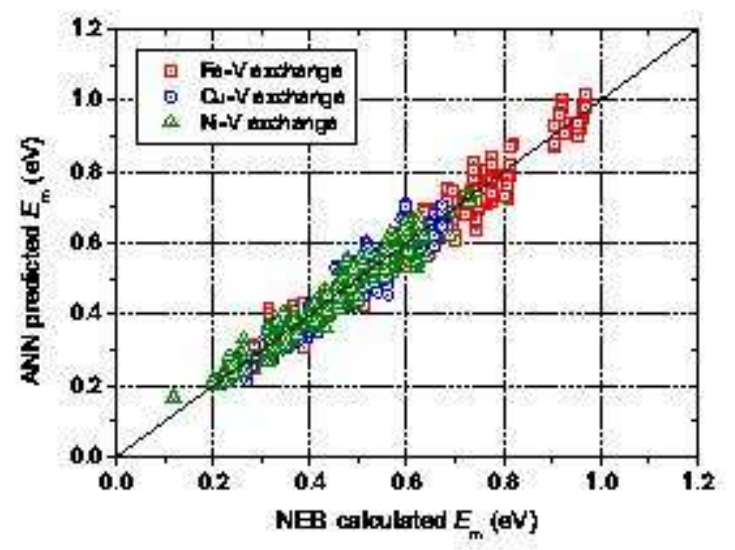

$106 \times 83 \mathrm{~mm}(72 \times 72$ DPI $)$ 